\title{
Antiferromagnetic Exchange Interactions from Hybrid Density Functional Theory
}

\author{
Richard L. Martin ${ }^{1}$ and Francesc Illas ${ }^{2}$ \\ ${ }^{1}$ Theoretical Division MS B268, Los Alamos National Laboratory, Los Alamos, New Mexico 87545 \\ ${ }^{2}$ Departament de Quimica Fisica, Universitat de Barcelona, C/Marti i Franques 1, 08028 Barcelona, Spain
}

(Received 27 March 1997)

\begin{abstract}
A hybrid theory which combines the full nonlocal "exact" exchange interaction with the local spindensity approximation of density-functional theory is shown to lead to marked improvement in the description of antiferromagnetically coupled systems. Semiquantitative agreement with experiment is found for the magnitude of the coupling constant in $\mathrm{La}_{2} \mathrm{CuO}_{4}, \mathrm{KNiF}_{3}$, and $\mathrm{K}_{2} \mathrm{NiF}_{4}$. The magnitude of the unpaired spin population on the metal site is in excellent agreement with experiment for $\mathrm{La}_{2} \mathrm{CuO}_{4}$. [S0031-9007(97)03942-2]

PACS numbers: 71.15.Mb, 75.10.Jm
\end{abstract}

The "local spin-density approximation" (LSDA) of density-functional theory (DFT) has proven extremely useful for understanding and predicting the electronic properties of condensed matter [1-4]. Despite its many successes, there are situations where the LSDA is known to give qualitatively incorrect descriptions; notable among these are those instances where the bands are narrow and the electrons nearly localized. In antiferromagnetic insulators such as $\mathrm{La}_{2} \mathrm{CuO}_{4}$ the LSDA exaggerates delocalization, thereby predicting the material to be a metal [5], or at least significantly underestimating the magnitude of the magnetic moment [6]. A number of remedies have been investigated, among them the self-interaction correction (SIC) [7] and LDA + U [8] approaches. In this Letter we examine an alternative; a hybrid functional which combines the full nonlocal "exact" exchange interaction with the exchange-correlation functional of the LSDA. We find that it qualitatively and semiquantitatively corrects this deficiency of conventional DFT for three representative antiferromagnets. Although the focus in this work is on cluster models, this DFT approach is amenable to implementation with periodic boundary conditions.

Hybrid density functionals [9-12] combine elements of Hartree-Fock (HF) theory with DFT, and have enjoyed significant success in the theoretical chemistry community, particularly in the prediction of molecular heats of formation and bond energies, where they often correct the LSDA tendency to overestimate molecular binding energies. In order to motivate the relevance of hybrid functionals for the localization/delocalization problem, we briefly introduce their formal justification. The conceptual foundation of DFT is that the ground-state density suffices to determine all the properties of a many-body system. In particular, the energy is given by

$$
E[\rho]=\int \rho(r) V_{\mathrm{ext}}(r) d r+T[\rho]+V_{e e}[\rho],
$$

where $E$ is the total energy of the fully interacting system of electrons, the integral over the external potential is the nuclear-electron attraction, and $T$ and $V_{e e}$ are functionals of the electron-density $\rho$ which describe the kinetic energy and electron-electron interactions. In the LSDA, $\rho$ is understood to generalize to $\rho_{\alpha}$ and $\rho_{\beta}$ spin densities. The ground-state density is that which minimizes the total energy.

In the Kohn-Sham formulation, the unknown kinetic energy functional is finessed by defining a fictitious noninteracting reference system described by a single determinant of orbitals $\left(\psi_{i}\right)$ whose density is identical to the ground-state density. The energy is partitioned

$$
E=\int \rho V_{\mathrm{ext}} d r+T_{0}+J[\rho]+E_{\mathrm{xc}}[\rho],
$$

where $E$ and the electron-nuclear term are as before, but $T_{0}$ is now the kinetic energy of the noninteracting determinant

$$
T_{0}=\sum_{i=1}^{N}\left\langle\psi_{i}\left|-\frac{1}{2} \nabla^{2}\right| \psi_{i}\right\rangle .
$$

Note also that the Hartree energy $J[\rho]$ has been segregated in Eq. (2) from the electron-electron energy and everything else is lumped into the exchange-correlation energy functional $E_{\mathrm{xc}}[\rho]$. In particular, corrections to the noninteracting kinetic energy are also found in $E_{\mathrm{xc}}[\rho]$. The Kohn-Sham orbitals are found by solving the equations

$$
\left(-1 / 2 \nabla^{2}+V_{0}\right) \psi_{i}=\epsilon_{i} \psi_{i},
$$

where the potential $V_{0}$ is given by

$$
V_{0}=V_{\mathrm{ext}}+V_{J}+V_{\mathrm{xc}}
$$

with $V_{J}$ the Hartree potential and $V_{\mathrm{xc}}=\delta E_{\mathrm{xc}} / \delta \rho$. Local (LSDA) and generalized gradient approximations (GGA) [13] to the exchange-correlation energy can be extracted from the electron gas problem or other solvable model systems such as the He atom [14].

Becke [9] has argued that improved functionals may be generated by consideration of the adiabatic connection formula [15-18]. The price paid for simplifying the kinetic energy through the introduction of a fictitious 
noninteracting system is that the exchange-correlation energy is given by an integral

$$
E_{\mathrm{xc}}=\int_{0}^{1} d \lambda U_{\mathrm{xc}}^{\lambda}
$$

where $\lambda$ is a coupling-strength parameter which turns on the Coulomb repulsion between electrons, and $U_{\mathrm{xc}}^{\lambda}$ is the potential energy of exchange correlation at coupling strength $\lambda$. It is understood that the density used to generate the exchange-correlation energy for all values of the coupling strength is fixed at the density of the real, fully interacting system. Becke suggests that it is the noninteracting $\lambda=0$ behavior of the LSDA which is physically inappropriate and responsible for its tendency to overbind molecular species. In particular, in that limit there is no correlation, only an exchange term, and it is the exact exchange energy of the single determinant of Kohn-Sham orbitals, call it $E_{x}$. If the Kohn-Sham orbitals were identical to the HF orbitals, this would be just the $\mathrm{HF}$ exchange energy. The form of the exchange hole in this limit is also well known. Consider, for example, $\mathrm{H}_{2}$ at its equilibrium distance. The $\lambda=0$ exchange hole is simply the negative of the $\sigma_{g}$ orbital density. It is static, delocalized over both centers, and provides the selfinteraction correction to the Hartree term. In contrast, the LSDA or GGA exchange holes are centered locally or semilocally about each electron and are dynamic.

In the present context, note that the simplest example of a narrow band system is realized by $\mathrm{H}_{2}$ as the bond length increases. At larger distances the electrons become more weakly interacting, and one might expect the $\lambda=0$ behavior of the functional to be even more important. The unphysical behavior of local approximations in this limit might be expected to become particularly apparent.

Less is known about the fully interacting limit, but presumably a local or gradient corrected approximation is reasonable. Suppose we assume the LSDA is sufficient in this limit. The simplest two-point approximation to the integral [Eq. (6)] is then

$$
E_{\mathrm{xc}}=\frac{1}{2}\left(E_{x}+U_{\mathrm{xc}, \lambda=1}^{\mathrm{LSDA}}\right) .
$$

The functional derivative may be used to construct an operator [Eq. (5)] and an associated set of orbitals and density obtained self-consistently. Becke denotes this the half-and-half method. This approach has been discussed and generalized recently by Levy, March, and Handy [19].

In the present work we take a similar, but distinct, approach. Separating the exchange-correlation potential energy in Eq. (6) into its components, we have

$$
E_{\mathrm{xc}}=\int_{0}^{1} d \lambda U_{x}^{\lambda}+\int_{0}^{1} d \lambda U_{c}^{\lambda} .
$$

Applying a two-point quadrature as before to the exchange term, but recognizing that the second integral is just the correlation energy, we have

$$
E_{\mathrm{xc}}=\frac{1}{2}\left(E_{x}+E_{x}^{\mathrm{LSDA}}\right)+E_{c}^{\mathrm{LSDA}} .
$$

Other hybrids can be defined by using GGA models, or with local approximations as above for the exchange, and a GGA for the correlation component. We use the notation F-S:VWN to refer to the approximation above, signifying that the exchange component is an equal mixture of exact (Fock) exchange and the local density (Slater) exchange, whereas the correlation component is the Vosko, Wilk, Nusair fit to the Ceperly-Alder homogenous electron gas correlation energy.

We have performed unrestricted Hartree-Fock (UHF), LSDA, GGA, and hybrid F-S:VWN calculations on cluster models of three representative antiferromagnets: $\mathrm{La}_{2} \mathrm{CuO}_{4}$, $\mathrm{KNiF}_{3}$, and $\mathrm{K}_{2} \mathrm{NiF}_{4}$. The first is a spin $1 / 2$ system, while the second and third are spin 1 on each nickel. The densities were determined by spin-unrestricted calculations on the clusters $\mathrm{Cu}_{2} \mathrm{O}_{11}$ and $\mathrm{Ni}_{2} \mathrm{~F}_{11}$. The primary clusters are embedded in a Madelung / Pauli background potential which reproduces the infinite lattice Madelung potential in the primary region as well as enforcing Pauli orthogonality between the electrons of the primary cluster and the first neighbor shell of the background $[20,21]$. The orbitals are expanded in standard double-zeta plus polarization basis sets. The coupling constant appropriate for the Heisenberg Hamiltonian is extracted from spin-unrestricted calculations, whose solutions are not pure eigenfunctions of the total spin operator, as discussed by Noodleman [22]. In brief, separate calculations on the high spin state and the unrestricted $S_{z}=0$ spin state are used to infer $J$. In the $\mathrm{Cu}$ dimer, for example, the magnitude of $J$ is given by the difference in energy between the triplet and singlet states. The spin-unrestricted $M_{s}=0$ single determinant is $\phi_{l} \phi_{r}[\alpha \beta]$, where $\phi_{l}$ and $\phi_{r}$ refer to molecular orbitals largely localized on the left and right sites of the dimer. This determinant is an equal superposition of the singlet wave function $\phi_{l} \phi_{r}[\alpha \beta-\beta \alpha]$, and the $M_{s}=0$ component of the triplet $\phi_{l} \phi_{r}[\alpha \beta+\beta \alpha]$. The difference in energy computed for the $\left(M_{s}=1\right)$ triplet state and the $M_{s}=0$ broken-symmetry determinant is then half the actual singlet-triplet splitting.

Finally, we must address how well the Heisenberg constants extracted from cluster calculations relate to those appropriate to the infinite lattice. Earlier research has shown that the superexchange interaction converges quickly with cluster size, and that it is possible to extract a reasonable approximation to $J$ in $\mathrm{La}_{2} \mathrm{CuO}_{4}$ (within $\sim 20 \%$ ) from a simple dimer containing only two metal sites [20,21]. Even more direct evidence for this conclusion was obtained in the present work, where UHF results for $\mathrm{Ni}_{2} \mathrm{~F}_{11}$ in a background potential appropriate for $\mathrm{KNiF}_{3}$ can be compared with UHF calculations on $\mathrm{KNiF}_{3}$ which employ fully periodic boundary conditions [23]. Similar basis sets were used in both calculations. The dimer model gives $J=2.58 \mathrm{meV}$, while the fully periodic calculations give $J=2.57 \mathrm{meV}$. Thus 
we believe that qualitative conclusions drawn from the clusters will transfer straightforwardly to fully periodic calculations.

Our results are presented in Table I. We focus on two parameters: the magnitude of $J$, and the "magnetic moment" as determined by the Mulliken unpaired spin population on the metal site in the unrestricted $\left(S_{z}=0\right)$ solution. Consider first the $\mathrm{Cu}_{2} \mathrm{O}_{11}$ results. The UHF unpaired spin population $\left(P_{d}\right)$ on the $\mathrm{Cu}$ sites is 0.90 , indicating a sizable moment. The remainder of the unpaired spin is delocalized onto the oxygen $2 p_{\sigma}$ orbitals. The localization of the moment is in fact overemphasized, the unpaired population being greater than the value of 0.80 inferred from experiment [24]. The UHF value for $J(38 \mathrm{meV})$ underestimates experiment $(J=128 \mathrm{meV})$ [25] by nearly a factor of 3 . The reason for this is understood. If the cluster were approximated by a single-band Hubbard Hamiltonian, then $J \sim t^{2} / U$, where $t$ is the effective hopping integral between the two metal sites, and $U$ is the on-site Coulomb repulsion. The HF approximation grossly overestimates $U$, thereby underestimating $J$. Acceptable agreement with experiment may be obtained through configuration-interaction (CI) expansions which explicitly screen $U$ to its proper value [20,21].

In contrast, the LSDA (S:VWN) underestimates the localization. As can be seen in Table I, it gives too little unpaired spin population on the metal sites $\left(P_{d}=\right.$ 0.31). Note that in the limit in which there is no spin polarization at all, the singlet-triplet energy difference is simply $2 t$, where $t$ is the effective hopping integral. Thus, the large energy difference in the LSDA, $J=820$ $\mathrm{meV}$, is more a measure of the effective hopping integral than an antiferromagnetic coupling constant. While early investigations of the periodic LSDA gave no sign of a spin-polarized solution [5], we note that Kasowski et al. [6] argue that if sufficient care is given to the representation of the potential, a spin-polarized solution is found with $P_{d}=0.35$, in good agreement with the value determined for the cluster.

An extension to a generalized gradient approximation is reported in the column labeled B:PW91. Here Becke's gradient-corrected exchange functional [27] is coupled with the gradient-corrected correlation functional of Perdew and Wang [28]. This approximation is a step in the right direction, but the unpaired population (0.49) is still too small, and the splitting $(601 \mathrm{meV})$ much too large. Most of the change from the LSDA result is associated with the exchange functional. For example, if only the exchange functional is replaced by a GGA $(\mathrm{B}: \mathrm{VWN})$, the unpaired population and coupling constants $(0.48$ and $610 \mathrm{meV}$ ) are nearly identical to B:PW91. Finally, the simple hybrid functional [Eq. (9)] is reported in the column labeled F-S:VWN. The agreement with experiment is much improved. The moment $\left(P_{d}=0.79\right)$ is in excellent agreement with experiment, and the magnitude of $J$ $(105.0 \mathrm{meV})$ is within $20 \%$ of experiment.

As additional tests, we examined the "locally" spin 1 systems $\mathrm{KNiF}_{3}$ and $\mathrm{K}_{2} \mathrm{NiF}_{4}$ (Table I). The general behavior exhibited by $\mathrm{La}_{2} \mathrm{CuO}_{4}$ is also evident here, although the LSDA gives a significant spin population in these cases. The magnitudes of $J$ predicted by the hybrid approach are $7.5 \mathrm{vs} 8.6 \mathrm{meV}$ (experiment) [26] for $\mathrm{K}_{2} \mathrm{NiF}_{4}$, and 7.0 vs $8.2 \mathrm{meV}$ (experiment) [26] for $\mathrm{KNiF}_{3}$. Once again, the theoretical splittings are smaller than experiment by about $20 \%$, but from a more general perspective the agreement in these cases is remarkable considering the interaction is only of the order of $100 \mathrm{~K}$. As regards the spin population, the hybrid functional again enhances localization on the metal site, but to our knowledge there are no experimental results with which to compare.

The results in Table I suggest that it is the unphysical nature of local or semilocal approximations for the exchange operator in the weakly interacting limit which is responsible for the problems of the LSDA in narrow band systems. This point is reinforced by the study of the additional hybrid combinations reported in Table II. The results are found to be largely insensitive to the correlation functional (LSDA vs GGA) employed in Eq. (9), and only marginally sensitive to the use of a GGA in the exchange expression. In fact, carrying the former observation to its logical conclusion, we also report a hybrid which ignores the correlation functional completely (F-S:null). It is, surprisingly, in general agreement with the other results. It is important to remember that $J$ is determined from energy differences, and we certainly do

TABLE I. The coupling constants $(J)$ and associated unpaired spin population $\left(P_{d}\right)$ on the metal site for the cluster models of $\mathrm{La}_{2} \mathrm{CuO}_{4}, \mathrm{~K}_{2} \mathrm{NiF}_{4}$, and $\mathrm{KNiF}_{3}$. The columns refer to unrestricted Hartree-Fock (UHF), local spin-density (S:VWN), gradientcorrected exchange and correlation (B:PW91), gradient-corrected exchange only (B:VWN), and hybrid (F-S:VWN) approximations, where the notation (exchange:correlation) and the specific functionals are described in the text.

\begin{tabular}{|c|c|c|c|c|c|c|c|}
\hline & & UHF & S:VWN & B:PW91 & B:VWN & F-S:VWN & Expt. \\
\hline \multirow[t]{2}{*}{$\mathrm{La}_{2} \mathrm{CuO}_{4}$} & $J(\mathrm{meV})$ & 37.8 & 820.0 & 601.0 & 610.3 & 105.0 & $128.0 \pm 0.7$ \\
\hline & $P_{d}$ & 0.90 & 0.31 & 0.49 & 0.48 & 0.79 & 0.8 \\
\hline \multirow[t]{2}{*}{$\mathrm{K}_{2} \mathrm{NiF}_{4}$} & $J(\mathrm{meV})$ & 2.9 & 57.1 & 41.3 & 38.9 & 7.5 & $8.6 \pm 0.4$ \\
\hline & $P_{d}$ & 1.92 & 1.61 & 1.68 & 1.68 & 1.86 & $\ldots$ \\
\hline \multirow[t]{2}{*}{$\mathrm{KNiF}_{3}$} & $J(\mathrm{meV})$ & 2.6 & 53.1 & 38.6 & 36.5 & 7.0 & $8.2 \pm 0.6$ \\
\hline & $P_{d}$ & 1.93 & 1.63 & 1.69 & 1.69 & 1.87 & $\cdots$ \\
\hline
\end{tabular}


TABLE II. The coupling constants $(J)$ and associated unpaired spin population $\left(P_{d}\right)$ on the metal site for the cluster models of $\mathrm{La}_{2} \mathrm{CuO}_{4}, \mathrm{~K}_{2} \mathrm{NiF}_{4}$, and $\mathrm{KNiF}_{3}$. The columns refer to various combinations of exchange and correlation functionals used with the hybrid expression in the text. LYP refers to the gradient-corrected correlation functional of Lee, Yang, and Parr.

\begin{tabular}{cccccccc}
\hline \hline & & F-S:VWN & F-B:VWN & F-S:LYP & F-B:LYP & F-S:null & Expt. \\
\hline $\mathrm{La}_{2} \mathrm{CuO}_{4}$ & $J(\mathrm{meV})$ & 105.0 & 98.8 & 110.7 & 101.1 & 101.9 & $128.0 \pm 0.7$ \\
& $P_{d}$ & 0.79 & 0.80 & 0.79 & 0.80 & 0.80 & 0.8 \\
$\mathrm{~K}_{2} \mathrm{NiF}_{4}$ & $J(\mathrm{meV})$ & 7.5 & 6.5 & 8.0 & 7.1 & 6.9 & $8.6 \pm 0.4$ \\
& $P_{d}$ & 1.86 & 1.87 & 1.85 & 1.86 & 1.86 & $\ldots .7$ \\
$\mathrm{KNiF}_{3}$ & $J(\mathrm{meV})$ & 7.0 & 6.1 & 7.4 & 6.6 & $8.2 \pm 0.6$ \\
& $P_{d}$ & 1.87 & 1.87 & 1.86 & 1.87 & 1.87 \\
\hline \hline
\end{tabular}

not mean to imply that the correlation functional is not important for other properties. We interpret this behavior as a reflection that local or semilocal correlation approximations should give similar results for both the triplet and spin-unrestricted $\left(S_{z}=0\right)$ densities when the moments are largely localized on the metal sites. In contrast, both the moment and the coupling constant are fairly sensitive to the fraction of Fock exchange incorporated in the operator [29]. For example, a popular empirical hybrid functional (B3LYP [30]) which combines $\sim 20 \%$ exact exchange with the B:LYP GGA yields $P_{d}=0.68$ and $J=237 \mathrm{meV}$ for $\mathrm{La}_{2} \mathrm{CuO}_{4}$, an error of nearly $100 \%$ for $J$. Still, this is a marked improvement over either the LSDA or GGA result, and the point we wish to make is a qualitative one. We conclude that hybrid DFT methods and their refinements hold promise for the study of condensed matter systems.

This work was sponsored by the U.S. Department of Energy through the LDRD program at Los Alamos, and the Comission Interministerial de Ciencia y Technologia of the Spanish Ministerio de Educacion y Ciencia, Project No. PB95-0847-C02-01. We thank P. J. Hay for generating the $\mathrm{Ni}^{2+}$ effective core potential used in the Madelung/Pauli background potential. We also thank Professor Axel Becke and Professor Mel Levy for helpful conversations.

[1] P. Hohenberg and W. Kohn, Phys. Rev. 136, B864 (1964).

[2] W. Kohn and L. J. Sham, Phys. Rev. 140, A1133 (1965).

[3] R. G. Parr and W. Yang, Density-Functional Theory of Atoms and Molecules (Oxford University Press, New York, 1989).

[4] R.M. Dreizler and E. K. U. Gross, Density Functional Theory: An Approach to the Quantum Many-Body Problem (Springer, Berlin, 1990).

[5] W. E. Pickett, Rev. Mod. Phys. 61, 433 (1989).

[6] R. V. Kasowski, M. H. Tsai, and J. D. Dow, Phys. Rev. B 41, 7744 (1990).

[7] A. Svane, Phys. Rev. Lett. 68, 1900 (1992); A. Svane and O. Gunnarsson, Phys. Rev. Lett. 65, 1148 (1990).

[8] M. M. Steiner, R. C. Albers, and L. J. Sham, Phys. Rev. B 45, 13272 (1992); V.I. Anisomov, M. A. Korotin, J. A. Zaanen, and O. K. Anderson, Phys. Rev. Lett. 68, 345
(1992); M. T. Czyzyk and G. A. Sawatzsky, Phys. Rev. B 49, 14211 (1994); P. Wei and Z. Q. Qi, Phys. Rev. B 49, 12519 (1994).

[9] A. D. Becke, J. Chem. Phys. 98, 1372 (1993).

[10] A. D. Becke, in Modern Electronic Theory, Part II, edited by D. A. Yarkony (World Scientific, Singapore, 1995).

[11] J. P. Perdew, M. Ernzerhof, and K. Burke, J. Chem. Phys. 105, 9982 (1997).

[12] A. Gorling and M. Levy, J. Chem. Phys. 106, 2675 (1997).

[13] J. P. Perdew, K. Burke, and M. Ernzerhof, Phys. Rev. Lett. 77, 3865 (1996).

[14] R. Colle and O. Salvetti, J. Chem. Phys. 79, 1404 (1983); C. Lee, W. Yang, and R. G. Parr, Phys. Rev. B 37, 785 (1988).

[15] J. Harris and R. O. Jones, J. Phys. F 4, 1170 (1974).

[16] O. Gunnarsson and B. I. Lundqvist, Phys. Rev. B 13, 4274 (1976).

[17] D. C. Langreth and J. P. Perdew, Phys. Rev. B 15, 2884 (1977).

[18] J. Harris, Phys. Rev. A 29, 1648 (1984).

[19] M. Levy, N. March, and N. Handy, J. Chem. Phys. 104, 1989 (1996).

[20] R. L. Martin and P. J. Hay, J. Chem. Phys. 98, 8680 (1993); R. L. Martin, J. Chem. Phys. 98, 8691 (1993).

[21] I. de P.R. Moreira and F. Illas, Phys. Rev. B 55, 4129 (1997); J. Casanovas, J. Rubio, and F. Illas, Phys. Rev. B 53, 945 (1996); A. B. Van Oosten, R. Broer, and W. C. Nieuwpoort, Chem. Phys. Lett. 257, 207 (1996).

[22] L. Noodleman, J. Chem. Phys. 74, 5737 (1981); L. Noodleman and E. R. Davidson, Chem. Phys. 109, 131 (1986).

[23] J. M. Roetti, R. Dovesi, C. Roetti, and V.R. Saunders, Phys. Rev. B 52, 2381 (1995).

[24] This value is inferred from the observed moment of $0.5 \mu_{B}$ using a reduction due to quantum fluctuations of 0.64 .

[25] G. Aeppli, et al., Phys. Rev. Lett. 62, 2052 (1989); Y. Endoh et al., Phys. Rev. B 37, 7443 (1988); K. Yamada et al., Phys. Rev. B 40, 4557 (1989).

[26] M.E. Lines, Phys. Rev. 164, 736 (1967); L. J. de Jongh and R. Block, Physica (Amsterdam) 79B, 568 (1975); L. J. de Jongh and R. Miedema, Adv. Phys. 23, 1 (1979).

[27] A. D. Becke, Phys. Rev. A 38, 3098 (1988).

[28] J. P. Perdew and Y. Wang, Phys. Rev. B 45, 13244 (1992).

[29] F. Illas and R. L. Martin (to be published).

[30] M. J. Frisch et al., GAUSSIAN94 (Gaussian Inc, Pittsburgh, PA, 1995). 